\title{
Fourier transforms in classical optics
}

\section{Chandrasekhar Roychoudhuri}

Chandrasekhar Roychoudhuri, "Fourier transforms in classical optics," Proc. SPIE 9663, Eighth International Topical Meeting on Education and Training in Optics and Photonics, 96632N (6 October 2003); doi: 10.1117/12.2207472

SPIE Event: Eighth International Topical Meeting on Education and Training in Optics and Photonics, 2003, Tucson, Arizona, United States 


\title{
FOURIER TRANSFORMS IN CLASSICAL OPTICS Chandrasekhar Roychoudhuri [Chandra@phys.uconn.edu] Photonics Lab., Physics department, University of Connecticut
}

\begin{abstract}
The principle of superposition plays critical roles in both classical and quantum physics. This paper will underscore the conceptual continuities and divergences in the process of successfully using Fourier transforms as a classical superposition tool to quantify Fraunhofer diffractions, far-field space and temporal coherence, Fourier transform spectroscopy and dispersive broadening of a short pulse.

\section{INTRODUCTION}

In physics, the principle of superposition in a linear domain and the Fourier mathematics have become synonymous by virtue of their great success in explaining old physics, predicting new physics and paving the way for a wide variety of technological break through (practical instrumentation). This article will focus on application of Fourier transform (\& series) in Optics and establish a conceptual continuity between the various phenomena that successfully use Fourier math.
\end{abstract}

\section{FRAUNHOFER DIFFRACTION}

This is one of the earliest example of identifying a mathematical representation of a physical phenomena based on a physical hypothesis to facilitate the understanding of the optical diffraction phenomenon. Huygens-Fresnel principle (or, HF integral) treats wave propagation as a superposition of secondary, spherical wavelets emanating out of every point on the wave front. This integral transforms into a superposition of plane waves in the far-field. Then the far-field integral resembles exactly like the Fourier transform integral of the near field illumination. This has given birth to the field of Fourier Optics for image processing. The detectable spatial energy distribution continuously evolves as the wave front propagates.

\subsection{Heuristic formulation of diffraction theory based on Huygens-Fresnel (HF) Principle.}

Every point on a wave front emits a new spherical wavelet, the "forward direction being normal to the original wave front point. The resulted new wave front due to propagation is the superposition of all the HF wavelet amplitudes at the target plane. In the case of our incoherent extended source, one only sums the intensities due to HF wavelets from every point of the incoherent source (Fig.1). A spherical wavelet originating at $\mathrm{O}$, has the phase delay (in radian) on the (one dimensional) X-plane given by $(2 \pi / \lambda) \cdot\left(\mathrm{x}^{2} / 2 \mathrm{r}\right)$. The exponential representation is $\exp (+\mathrm{ikx} / 2 \mathrm{r})(+$ sign convention for the point of divergence on the left).

\footnotetext{
Eighth International Topical Meeting on Education and Training in Optics and Photonics,

edited by Barry L. Shoop, Grover Swartzlander Jr., Proc. of SPIE Vol. 9663, 96632N

(c) 2003 SPIE, OSA, ICO · doi: 10.1117/12.2207472
} 


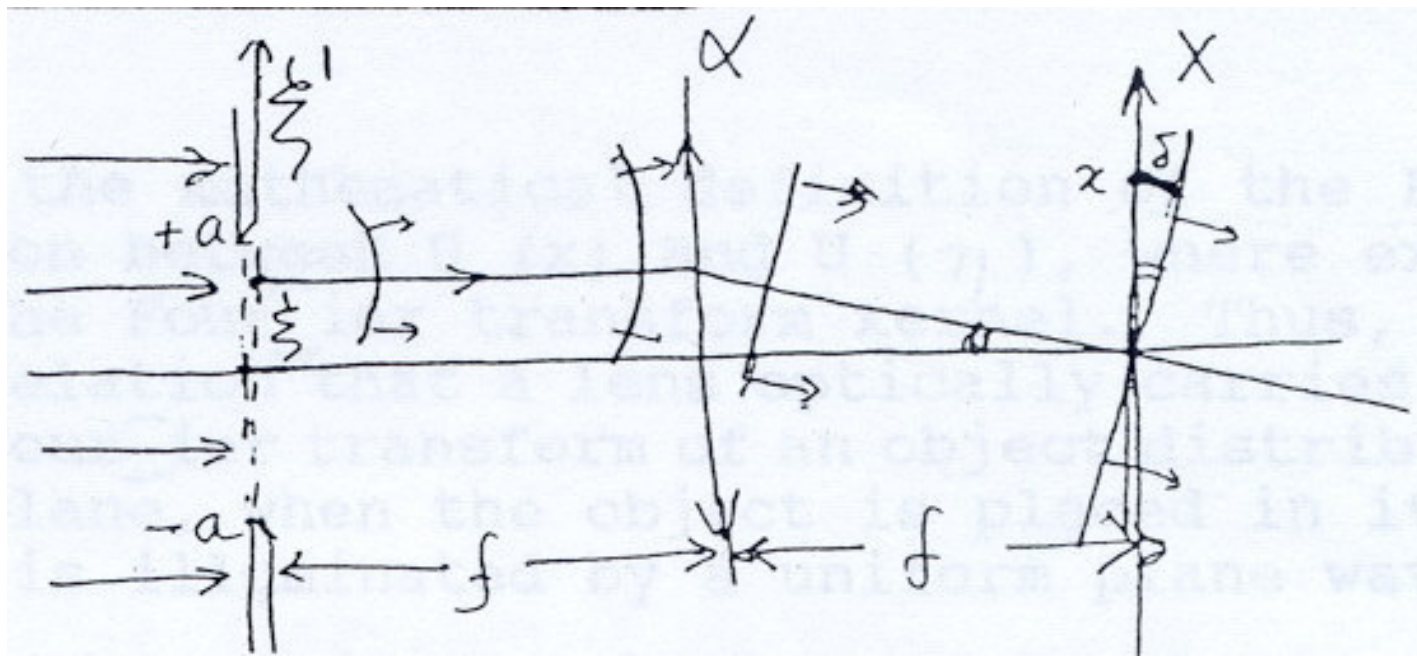

Figure 1. Heuristic derivation of Far-field diffraction pattern using simple geometrical and physical optics.

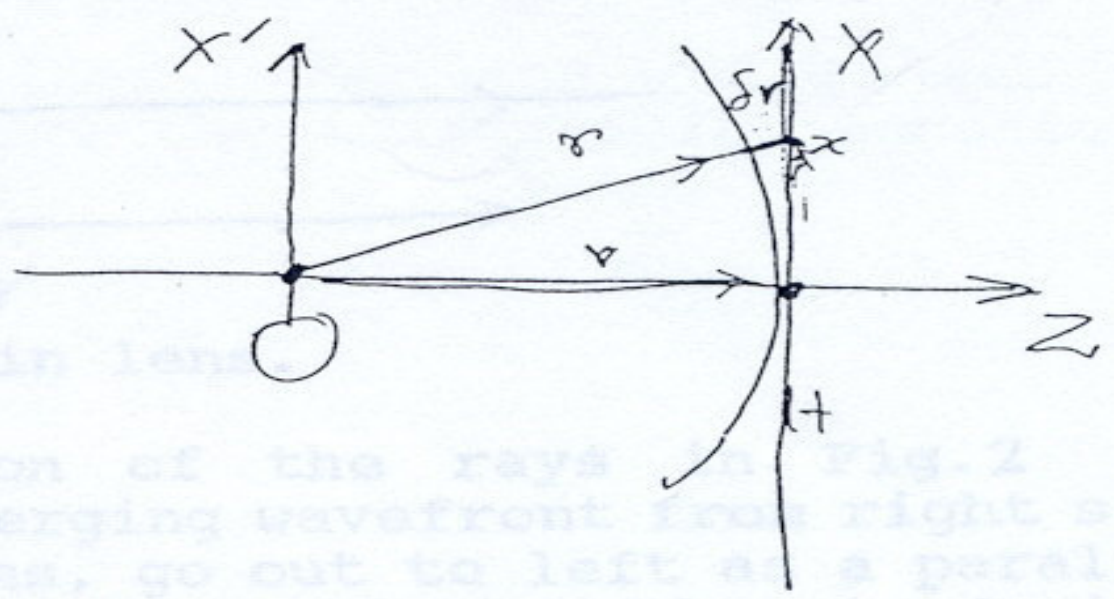

Figure 2. A spherical wave front, at small angle approximation, gives a phase front $\exp \left(+i k x^{2} / 2 r\right)$ on the planar $X$-axis.

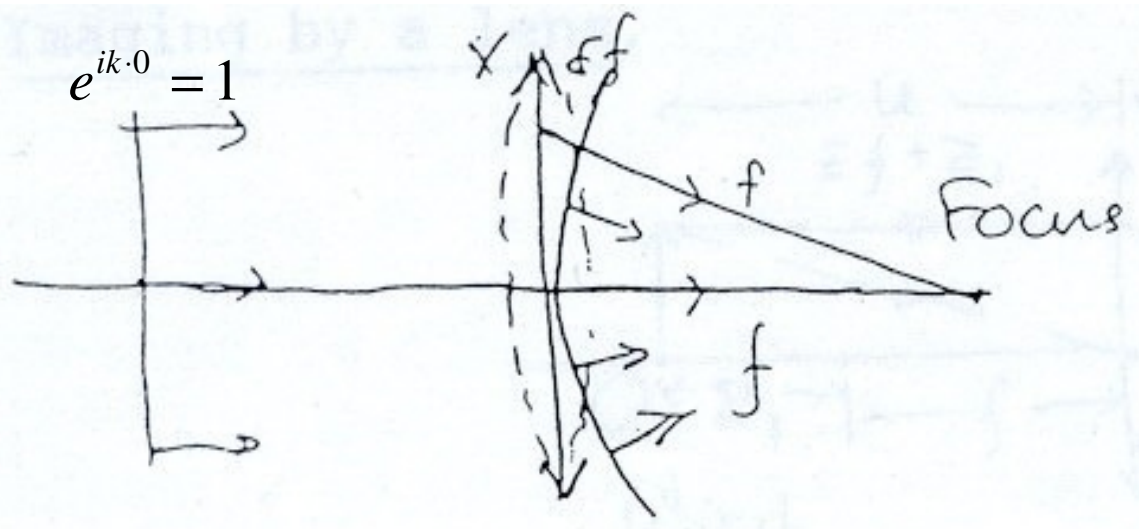

Figure 3. A lens introduces a phase factor $\exp \left[-\mathrm{ikx} x^{2} / 2 f\right]$. This causes a plane wave to converge at its back focal plane. 


$$
\begin{gathered}
\mathrm{x}^{2}+\mathrm{f}^{2}=(\mathrm{f}+\delta \mathrm{f})^{2}=\mathrm{f}^{2}+\delta \mathrm{f}^{2}+2 \mathrm{f} \delta \mathrm{f} \approx \mathrm{f}^{2}+2 \mathrm{f} \delta \mathrm{f}\left(\text { neglecting small } \delta \mathrm{f}^{2}\right) \\
\text { Or, } \delta \mathrm{f}=\left(\mathrm{x}^{2} / 2 \mathrm{f}\right)
\end{gathered}
$$

\section{A lens creates a Fourier transform in its focal plane:}

We want to find out the complex amplitude $\mathrm{U}(\mathrm{x})$ on the $\mathrm{x}$-plane (back focal plane) when an object $\mathrm{U}(\xi)$ is illuminated by a uniform, continuous wave $(\mathrm{CW})$, monochromatic (single carrier frequency), plane wave at the front focal plane ( $\xi$-plane). We will use the concepts of ray optics, wave optics, and Huygens-Fresnel principle along with the built-in principle of linear (amplitude) superposition of coherent waves.

Consider a general point, $\xi$, on the $\xi$-plane. It emanates a spherical HF wavelet $\&$ arrives at the lens- $(\alpha-)$ plane with a curvature $\exp \left[+i k \alpha^{2} / 2 \mathrm{f}\right]$. This quadratic curvature gets flattened by the reverse quadratic phase factor of the lens $\exp \left[\left[-i \mathrm{k} \alpha^{2} / 2 \mathrm{f}\right]\right.$ and a plane wave-let emanates toward the focal plane. The plane wavelet intersects the $\mathrm{x}$-plane at an angle $\theta=\sin ^{-1}(\delta / x) \approx \tan ^{-1}(\xi / f)$, where $\delta$ is the relative delay of the plane wavelet at the $\mathrm{x}$-point on the X-plane and $\theta$ is a small angle. So, the path delay is, $\delta=\xi x / f .$. Or, the titled plane wave representation is $\exp [\mathrm{ik} \xi \mathrm{x} / \mathrm{f}]$.

If the complex amplitude at the point of origin of the HF wavelet at the $\xi$-point is $\mathrm{U}_{\xi}(\xi)$, then the complex amplitude at the $\mathrm{x}$ point is $\mathrm{CU}_{\xi}(\xi) \exp [\mathrm{ik} \xi \mathrm{x} / \mathrm{f}]$, where $\mathrm{C}$ is a propagation constant including amplitude reduction due to spherical divergence.

The total contribution of complex amplitude at the $\mathrm{x}$ point, $\mathrm{U}_{\mathrm{x}}(\mathrm{x})$, is the linear superposition (sum, or integral) of all the contributing points from the $\xi$-plane, so the integration should cover the entire "open aperture":

$$
\begin{aligned}
\mathrm{U}_{\mathrm{x}}(\mathrm{x}) & =\mathrm{C} \int \mathrm{U}_{\xi}(\xi) \exp [\mathrm{ik} \xi \mathrm{x} / \mathrm{f}] \mathrm{d} \xi \\
\text { Or, } \mathrm{U}_{\mathrm{x}}(\mathrm{x}) & =\mathrm{C}^{\prime} \int \mathrm{U}_{\xi}\left(\xi^{\prime}\right) \exp \left[2 \pi \mathrm{i} \xi^{\prime} \mathrm{x}\right] \mathrm{d} \xi^{\prime}
\end{aligned}
$$

Where we have substituted $\xi^{\prime}=\xi / \lambda f$. This last integral equation is the mathematical definition of the Fourier transform relation between $\mathrm{U}_{\mathrm{x}}(\mathrm{x})$ and $\mathrm{U}_{\xi}\left(\xi^{\prime}\right)$.

Although the above derivation is carried out heuristically, without going through the rigorous (Raylength-Sommerfeld or other similar) formulation, it preserves all the fundamental concepts accurately except some constants that give the exact numerical value for the complex amplitude. The key point to note here is that the exponential factor within the integral looks like the mathematical Fourier transform kernel. Thus the far-field diffraction pattern is the Fourier transform of the diffracting aperture function. This fortunate mathematical coincidence paved the way for the development of a major field, optical signal processing, where Fourier mathematics and related theorems serve as major mathematical tools. One should note two important points here: 
(i) First, the appearance of the Fourier transform-like structure of the Eqns. $2 \& 3$ is an evolutionary consequence of the physical hypothesis (Huygens-Fresnel Principle) that a propagating wave front can be represented as a superposition of an infinite number of spherical wavelets.

(ii) Second, the Eqns. 3 \& 4 represent real physical propagation of light waves from one physical plane to another, allowing us the opportunity for optical signal processing by real physical manipulation of the diffracting wave fronts with different physical apertures.

\subsection{Rigorous Diffraction Theory.}

The rigorous Rayleigh-Sommerfeld formulation of diffraction theory based on HuygensFresnel principle is given by the Eq. 1 [Goodman, Eq.4-8]:

$$
\mathrm{U}\left(\mathrm{P}_{0}\right)=(1 / \mathrm{i} \lambda) \int \mathrm{U}\left(\mathrm{P}_{1}\right)\left[\exp \left(\mathrm{ikr}_{01}\right) / \mathrm{r}_{01}\right] \cos \theta \mathrm{ds}
$$

For a rectangular diffracting aperture, the far-field (or, Fraunhofer) diffraction pattern, with small angle approximation, the Eq.1 simplifies into a summation (integral) of plane waves:

$$
\mathrm{U}(\mathrm{x}, \mathrm{y})=\left[\exp (\mathrm{ikz}) \cdot \exp \left\{\mathrm{ik}\left(\mathrm{x}^{2}+\mathrm{y}^{2}\right) / 2 \mathrm{z}\right\} / \mathrm{iz} \lambda\right] \int \mathrm{U}(\xi, \eta) \exp \{-2 \pi \mathrm{i}(\mathrm{x} \xi+\mathrm{y} \eta) / \lambda \mathrm{z}\} \mathrm{d} \xi \mathrm{d} \eta
$$

For conceptual simplicity, let us consider the one dimensional case of an infinitely long diffracting slit. Ignoring some constants and phase factors outside the integral, and after substituting $(\xi / \lambda \mathrm{z})=\xi$, one can write:

$$
\mathrm{U}(\mathrm{x})=\mathrm{C}^{\prime} \int \mathrm{U}\left(\xi^{\prime}\right) \exp \left\{-2 \pi \mathrm{i} x \xi^{\prime}\right\} \mathrm{d} \xi^{\prime}
$$

The Eqns. (3), (4) and (6), (7) are equivalent.

2. Fourier Transform Spectroscopy: When radiated electromagnetic field (EMF) is split into two beams and is superposed again with a relative delay between them, one can record the detectable energy as sinusoidally varying intensity fringes, which we call interferograms. The spatial variation of these periodic fringes is exactly related to the carrier frequency of the EMF. When one has many such $\mathrm{CW}$ carrier frequencies, each frequency forms its own periodic fringes, because interferogram materials do not record time varying mutual interference between different carrier frequencies. It only records the time averaged, selfinterference (light beams of different frequencies do not interfere with each other). The resultant interferogram now has a variable visibility function, multiplied by an average cosine oscillatory component. Fourier mathematics also uses sinusoidal functions in its transformational relation. Thus, the oscillatory part of the interferogram can be "Fourier transformed" to extract the spectral energy distribution of the original complex EMF. This is at the foundation of the successful field of Fourier transform spectroscopy applied in understanding fundamental physics and chemistry of atoms and molecules. Thos field was 
developed by A. Michelson in late 1800's, which earned him a Nobel Prize, the first for a US physicist.

When a collimated monochromatic wave (CW or continuous wave carrying single frequency) passes through a Michelson interferometer with an asymmetric beam splitter (not a 50/50 one), the superposed amplitudes on the beam splitter can be represented by,

$$
E_{r}\left(t, v_{0}, \tau\right)=A_{1} \cos \left(2 \pi v_{0} t-\varphi_{1}\right)+A_{2} \cos \left(2 \pi v_{0} t-\varphi_{2}\right)
$$

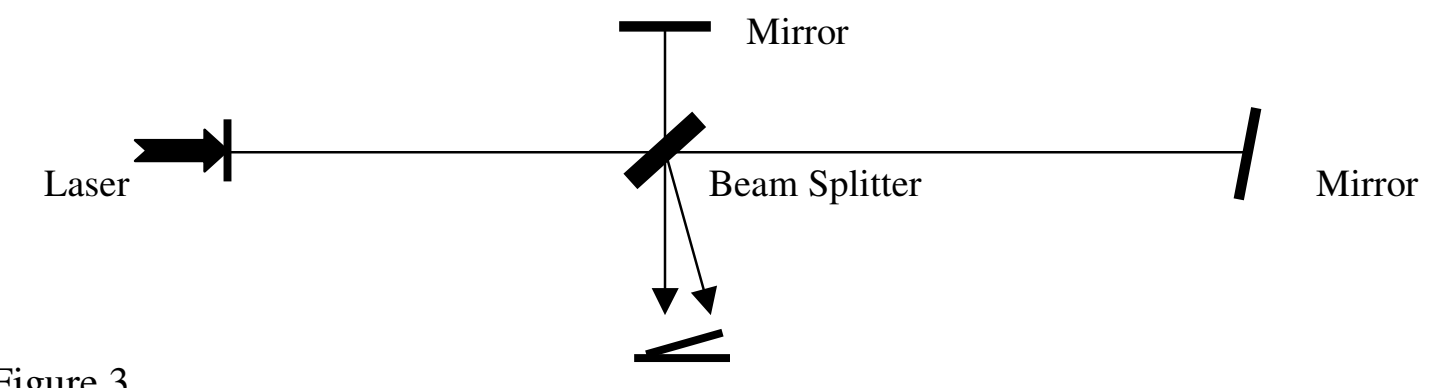

Figure 3. ....................

$\mathrm{E}_{\mathrm{r}}$ indicates real representation of E-field, instead of complex representation, used later. The relative phase delay between the two wave front is $\left(\varphi_{2}-\varphi_{1}\right)=2 \pi v_{0} \tau, \tau$ being the relative time delay (total path delay $\delta$ divided by c). We cannot detect the amplitude of light. We can only infer the presence of light by carefully observing light induced changes in material properties as it absorbs energy from the electromagnetic field. The light induced reaction (photochemical, photo-electric or photo-conductive), I, is proportional to time average of the square of the light amplitude [Klein]:

$$
\begin{aligned}
\mathrm{I}_{\mathrm{d}}\left(v_{0}, \tau\right) & =(1 / \mathrm{T}) \int \mathrm{E}_{\mathrm{r}}^{2}\left(\mathrm{t}, v_{0}, \tau\right) \mathrm{dt} \\
& \left.\approx \mathrm{I}_{1}+\mathrm{I}_{2}+2 \sqrt{ }\left(\mathrm{I}_{1} \mathrm{I}_{2}\right) \cos 2 \pi v \tau=\mathrm{I}_{0}+\mathrm{I}_{0} \mathrm{x} \cos 2 \pi v_{0} \tau\right),
\end{aligned}
$$

where, $\mathrm{I}_{0}=\left(\mathrm{I}_{1}+\mathrm{I}_{2}\right)$, and $\mathrm{x}=2 \sqrt{ }\left(\mathrm{I}_{1} \mathrm{I}_{2}\right) /\left(\mathrm{I}_{1}+\mathrm{I}_{2}\right)$,

and, $\quad \mathrm{I}_{\mathrm{osc}}=\mathrm{I}_{0} \times \cos 2 \pi v_{0} \tau=\mathrm{I}_{0} \times \cos 2 \pi \mathrm{m}$.

In the last step, we have used the order of interference, $m=(\delta / \lambda)=v_{0} \tau$. This $m$ is a very important parameter in all interferometry. In the linear domain, the source frequency remains unaltered, or a constant, so the location of the repeated, fringes for the same source frequency, is determined by $\tau$, given by $m=v_{0} \tau$. So, the variable component of the recorded fringe, $I_{0} \times \cos 2 \pi v_{0} \tau$, that is of importance for us. If $v_{0}$ is unknown, and the unequal beam amplitude factor, $x$ is fixed for a given beam splitter, then by counting the fringe $(\mathrm{m})$ with measured value of $\tau$, will give us the value of the carrier frequency, $v_{0}$, if the beam is monochromatic. But, we have a very complex interpretational problem if $\mathrm{x}$ is a variable and, especially, if it varies with time, either due to changing reflectivity of the beam splitter with time, or due to amplitude variation of the incident beam with time as produced by the source.

Let us consider the case of a time varying amplitude, or a pulsed source. Let us assume that we have cut out a Gaussian pulse from the above single frequency, CW laser, oscillating with a frequency, $v_{0}$. It actually could be a quite long pulse of width $\delta$, as long as the moving 
mirror of the Michelson interferometer can be moved by $\tau>\delta$ t. Now, we have a situation even when the beam splitter is a 50/50 divider, instead of $\mathrm{x}$ being unity, it still remains as an unequal beam amplitude factor, $x(t)$. Now the fringe visibility varies with time due to the unequal amplitudes incident on the beam splitter (and the detector) at different times, except when the delay, $\tau$, between the two mirror paths is exactly zero. By repeating the experiment with the same pulse but with varying values of the delay, $\tau$, we can obtain the variation of the fringe visibility with delay, as shown below. For each experiment with one pulse, one must integrate the interference signal for the entire duration of the two pulses (replicated, delayed and superposed by the Michelson interferometer). Note that we have only one source frequency, but a changing fringe visibility with $\tau$. One can mathematically show that the Fourier transform of the fringe visibility function (conjugate variables, $v_{\mathrm{f}}$ and $\tau$ ) is equal to the normalized, square modulus of the Fourier transform of the pulse amplitude function, $\mathrm{a}\left(v_{0}, \mathrm{t}\right)$ (conjugate variables being $v_{\mathrm{f}}$ and $\tau$ ). This is also called the Wiener-Khintchine theorem or the autocorrelation theorem [Klein]. We will come to it later again. We are underscoring the suffix of $v_{\mathrm{f}}$ as it represents mathematical variable "Fourier frequencies", in contrast to the frequency of the electric vector originating at the emitting source. Our point is that turning on a simple mechanical shutter to create amplitude pulse from a $\mathrm{CW}$, monochromatic source, does not create any new electric vector frequencies. This is a linear process. The fringe visibility varies for a pulsed light because of superposition of unequal amplitudes, and not due to new optical spectrum.

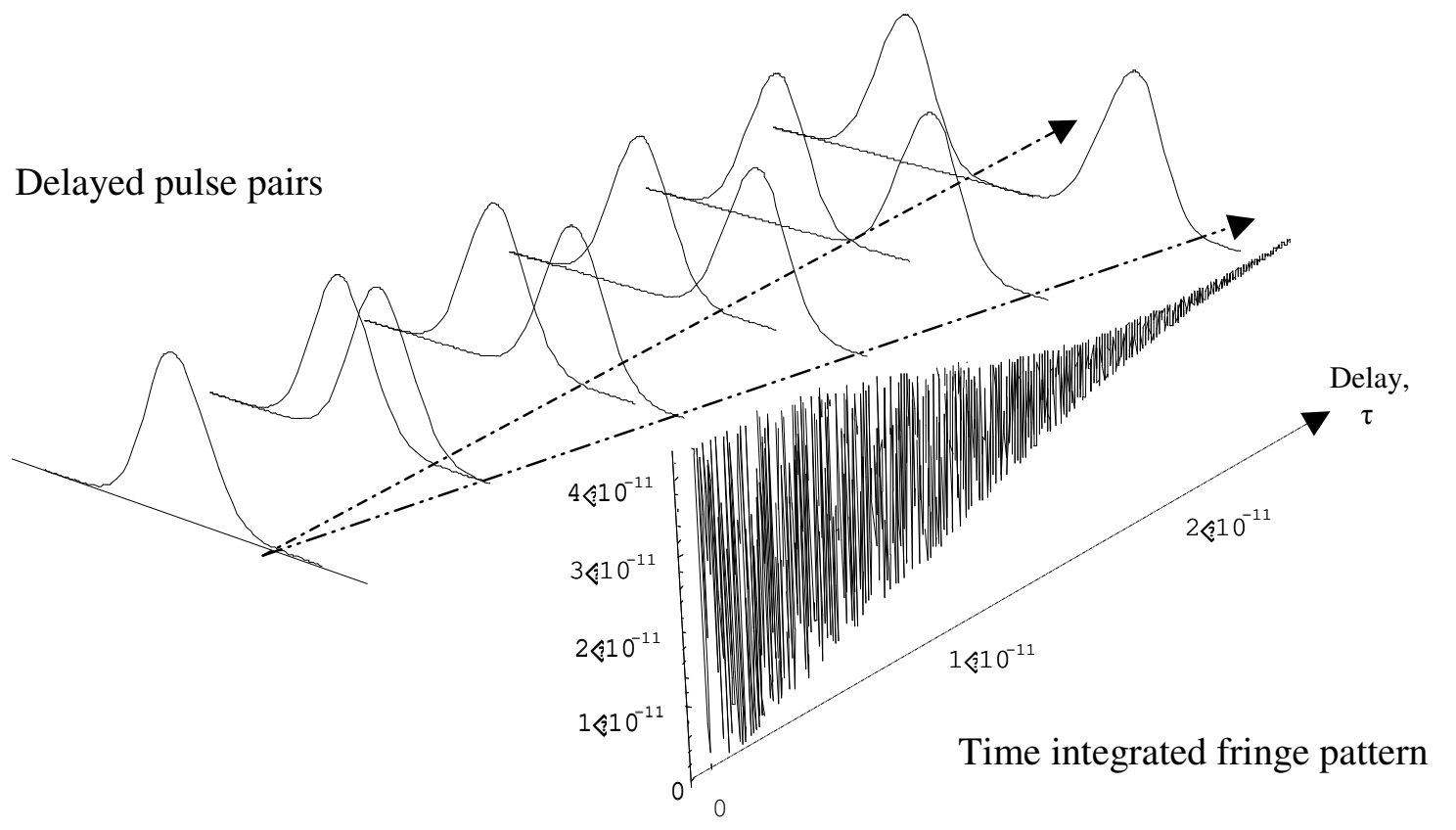

Figure 4. Variation of long-time integrated, fringe visibility in a simple Michelson interferometer as the delay between the mirrors are varied.

The situation is even more complex when the incident beam contains many unknown carrier (or source) frequencies. Let us now consider the case of a $\mathrm{CW}$ beam with two carrier 
frequencies, $v_{1}$ and $v_{2}$, and the narrow reflectivity coatings of the beam splitter gives different values for the unequal beam amplitude factor $\mathrm{x}$ as $\mathrm{x}_{1}$ and $\mathrm{x}_{2}$. Under this situation, Eq.(11) can be re-written as:

$$
\mathrm{I}_{\mathrm{osc}}=\mathrm{I}_{01} \mathrm{x}_{1} \cos 2 \pi v_{1} \tau+\mathrm{I}_{02} \mathrm{x}_{2} \cos 2 \pi v_{2} \tau
$$

There is a very simple and yet profoundly important, and experimentally validated assumption buried in Eq.(12), that different optical frequencies do not interfere with each other, as long as we use slow detector. Cross terms between different source frequencies have been dropped for slow detectors (see last section on beat and mode locking). If we use a $50 / 50$ beam splitter, the situation is a bit simpler with $\mathrm{x}_{1}=\mathrm{x}_{2}=1$, and one gets:

$$
\mathrm{I}_{\mathrm{osc}}=\mathrm{I}_{01}\left(v_{1}\right) \cos 2 \pi v_{1} \tau+\mathrm{I}_{02}\left(v_{2}\right) \cos 2 \pi v_{2} \tau
$$

If our goal is to determine the source frequencies, $v_{1}$ and $v_{2}$, we are still stuck with two more unknowns, $\mathrm{I}_{01}$ and $\mathrm{I}_{02}$. If these are equal, and let us normalize them, to unity, then we have:

$$
\mathrm{I}_{\mathrm{osc}}=\cos 2 \pi \nu_{1} \tau+\cos 2 \pi \nu_{2} \tau=2 \cos 2 \pi v_{\mathrm{s}} \tau \cdot \cos 2 \pi \nu_{\mathrm{d}} \tau
$$

Where, $v_{\mathrm{s}}=\left(v_{1}+v_{2}\right) / 2$ and $v_{\mathrm{d}}=\left(v_{1}-v_{2}\right) / 2$, are the mean of the sum and the mean of the difference of the two source frequencies. Thus, Eq.(14) describes the modulation of high frequency spatial fringes $\left(\mathrm{m}_{1,2}=v_{1,2} \tau\right)$ with a low frequency factor $v_{\mathrm{d}}=\left(v_{1}-v_{2}\right) / 2$, also known as waxing and waning of fringe visibility. In early times, people used this technique to determine $\left(v_{1}-v_{2}\right)$ of some atomic spectra, like Sodium fin structure doublet, known as $\mathrm{D}_{1}$ and $\mathrm{D}_{2}$ spectral lines. After the inventions of lasers and holography, people are innovating various techniques by exploiting this repeated oscillation of fringe visibility as a tool to accurately measure the depth and shape of complex 3D objects, etc. Eq.(14) can be generalized for the case of continuously distributed frequencies:

$$
\mathrm{I}_{\mathrm{osc}}=\int \mathrm{I}(v) \cos 2 \pi v \tau \mathrm{d} v
$$

The Fourier transformed, mathematical inversion relation is then given by [see Klein]:

$$
\mathrm{I}(v)=\int \mathrm{I}_{\mathrm{osc}}(\tau) \cos 2 \pi \mu \tau \mathrm{d} \tau
$$

As in Eqns (3), (4) or (6), (7) where the diffraction equations naturally evolves into a form that becomes identifiable with Fourier transform, here also the oscillatory part of an interferogram takes the form of a simple cosine Fourier transform. Just as in case of diffraction, the Fourier transform conjugate variables $(\xi-x)$ for space-space diffractive propagation, are real and based on validated Physics hypothesis (HF Principle), here also the conjugate variables $(v-\tau)$ are physically measurable quantities. However, the common root of identity of the mathematical expression of a physical phenomenon with a mathematical theorem arises from the linearity and the use of sinusoidal function as the basis. Electromagnetic field follows sinusoidal oscillation, and the basis function for Fourier transform theorem is also sinusoidal function. However, the Eq.(15) must still be used with great caution. First, it sums fringe intensities, and not field amplitudes, and ignores cross 
products between various source frequencies. However, as mentioned before, this is a valid assumption for slow, time averaging light detectors. Second, we have dropped $\mathrm{x}(\mathrm{v}, \mathrm{t})$ in Eq.(15) based on the assumption that all the source frequencies have sustained, CW amplitudes of $\sqrt{ } \mathrm{I}(\mathrm{v})$. If the atoms and molecules literally emit packets of spreading (according to HF Principle), classical light pulses, each carrying unique source frequencies, given by the quantum condition $\Delta \mathrm{E}_{\mathrm{n}}=\mathrm{h} v_{\mathrm{n}}$, then Michelson's spectroscopy should be generalized using Eq.8 instead of Eq.(11), with the added attention that the superposed amplitudes are functions of time. This is the model that is illustrated in Fig.4. If the incident pulse is a(t)exp[i2 $\left.\pi v_{0} t\right]$, then the complex version of Eq.(8) is given by:

$$
\mathrm{E}_{\mathrm{c}}\left(\mathrm{t}, v_{0}, \tau\right)=\mathrm{a}(\mathrm{t}) \exp \left[\mathrm{i} 2 \pi v_{0} \mathrm{t}\right]+\mathrm{a}(\mathrm{t}-\tau) \exp \left[\mathrm{i} 2 \pi v_{0}(\mathrm{t}-\tau)\right]
$$

Fast photo-induced energy exchange is equally well given by a time average integral of $\mathrm{E}_{\mathrm{r}}{ }^{2}$, as in Eq.(9) when the E-field is represented by real function, or simply by $\left(E_{c} E_{c}{ }^{*}\right)$, when $E_{c}$ is complex. As illustrated in Fig.4, the recorded fringe intensity needs a second time integration to cover the entire duration of the pair of pulses:

$$
\mathrm{I}_{\mathrm{d}}\left(v_{0}, \tau\right)=\int \mathrm{E}_{\mathrm{c}}\left(\mathrm{t}, v_{0}, \tau\right) \mathrm{E}_{\mathrm{c}}^{*}\left(\mathrm{t}, v_{0}, \tau\right) \mathrm{dt}
$$

After substitution of Eq.(17) in Eq.(18) and a series of simplification, one can obtain the oscillatory component of the fringe variation as:

$$
\mathrm{I}_{\mathrm{osc}}\left(v_{0}, \tau\right)=\gamma(\tau) \cdot \cos \left(2 \pi v_{0} \tau\right)
$$

Eq.(19) is good for single frequency, single pulse. This is the parallel of Eq.(11) for a single frequency, CW source. The function $\gamma(\tau)$ is the normalized auto correlation of the pulse a(t). The time integrated, oscillatory fringe for a Doppler broadened, thermal discharge lamp with atomic emitter can now be modeled as:

$$
\mathrm{I}_{\text {Dop. osc }}(\tau)=\int \mathrm{I}_{\mathrm{osc}}(\nu, \tau) \mathrm{d} v=\int \gamma(\tau) \cos 2 \pi v \tau \mathrm{d} \nu
$$

One can compare this with the Eq.(15) for the CW case. The RHS of Eq.(20) represents the mathematical Fourier transform of the autocorrelation function. By the autocorrelation, or Wiener-Kintchine theorem,

$$
\mathrm{S}_{\mathrm{f}}(v)=\int \gamma(\tau) \cos 2 \pi v \tau \mathrm{d} v
$$

Here $S_{f}(v)$ is the normalized, square modulus of the original pulse, a(t). Now, the Eqns.(20) and (21), implies that the oscillatory interferogram, $\mathrm{I}_{\text {Dop. osc }}(\tau)$, is equivalent to the mathematical spectral density function, $S_{\mathrm{f}}(v)$.

\section{BEAT SPECTROSCOPY AND MODE LOCKING}

In the above two examples of experimental success, the energy of the EMF is recorded over a time as an average of the square of the instantaneous amplitudes. Along with the hypothesis 
that in the time averaged recording, there is no interference between different frequencies. But, both beat spectroscopy and mode locking generate time varying energy "re-distribution" of superposed EMF of different frequencies. Then how does one reconcile the observational success of the time average recording of the first two phenomena (Far-field diffraction \& FT spectroscopy)? We have recently published a paper [Optics Express 11 (8), p.944, (2003)] that helps one to understand the conceptual continuity between all these phenomenon by simply accepting the hypothesis that the principle of superposition is manifest only in interacting materials, dictated by their quantum properties; EMF's do not operate on (or, change) each other. They simply pass through each other. The detailed discussions and experimental validations are presented in the above paper.

\section{ACKNOWLEDGEMENTS}

The author would like to gratefully acknowledge useful discussions with colleagues Masahiro Oikawa, Vladimir Serikov and Shigeo Kittaka of NSG Corporation. Some of the computations presented in the figures here, were computed by David Young and DongIk Lee.

\section{REFERENCES}

Klein, Miles V., "Optics".

Goodman, Joseph, "Introduction to Fourier Optics" 